\title{
Negative refraction and subwavelength focusing using photonic crystals
}

Ekmel Ozbay

Koray Aydin

K. B. Alici

Kaan Guven 


\title{
Negative refraction and subwavelength focusing using photonic crystals
}

\author{
E. Ozbay*a,b, K. Aydin ${ }^{\mathrm{b}}$, K. B. Alici ${ }^{\mathrm{b}}$, and K. Guven ${ }^{\mathrm{b}}$ \\ aAdvanced Research Laboratory, Bilkent University, 06800 Ankara, TURKEY \\ ${ }^{\mathrm{b}}$ Department of Physics, Bilkent University, 06800 Ankara, TURKEY
}

\begin{abstract}
We review certain novel optical properties of two-dimensional dielectric photonic crystals (PCs) which exhibit negative refraction behavior. We investigate two mechanisms which utilize the band structure of the PC and lead to a negative effective index of refraction $\left(n_{\text {eff }}<0\right)$. The negative refraction phenomenon is demonstrated experimentally and by simulations when the incident beam couples to a photonic band with $n_{\text {eff }}<0$. Further, the PC slab acts like a focusing lens to an omnidirectional source where the properties of focusing depends on the details of the band structure. In one case, by utilizing the TM polarized first band, an image of the source can be formed in the vicinity of the interface with subwavelength resolution. In another case, a TE polarized upper band is used which is able to focus the omnidirectional field far away from the interface with a resolution on par with the wavelength. In the latter case, we explicitly show the flat lens behavior of the structure. These examples indicate that PC based lenses can surpass limitations of conventional lenses and greatly enhance and extend optics applications.
\end{abstract}

Keywords: Photonic crystal; negative refraction; subwavelength resolution.

\section{INTRODUCTION}

Throughout the last decade, it has been a pleasing surprise to observe that photonic crystals (PCs), which are periodically modulated dielectric or metallic media, have been able to reveal so rich and novel electromagnetic (EM) phenomena despite their deceivingly simple structure. Their name is inherited from the analogy of the photon to an electron moving in a periodic electric potential in a solid. ${ }^{1}$ The periodicity essentially gives rise to bands for the propagation of electromagnetic waves, and band gaps, where the propagation is prohibited for certain frequency range. The band gaps an the cavity (defect) modes within rapidly found their way into applications in photonic devices. ${ }^{2}$ Using different materials (i.e., different dielectric constants), and by adjusting the geometrical parameters of the crystal, the band structure, and hence the propagation of light can be modified virtually in any way in a controllable manner. Moreover, the lack of confinement in a spatial direction does not hinder the investigation of essential features of the EM phenomena, hence one- or two-dimensional (2D) PC structures can be used in the first place, which are easier to fabricate compared to their 3D counterparts. The scalability of the governing Maxwell's equations enables the study to be conducted in an appropriate range of the electromagnetic spectrum (e. g., microwave), before extending it to the intended regime (infrared or optical). Evidently, the enormous application possibilities ${ }^{3-7}$ in telecommunication and optical wavelength scales drives the fabrication of PCs towards micro- and nanoscale. ${ }^{8,9}$

At first, most of the studies are concerned on utilizing the photonic band-gaps, band-edges, defect modes, and coupled cavity waveguides. ${ }^{2}$ However, the photonic crystals were hiding an easter egg: unusual dispersion characteristics of photonic bands can lead to anomalous refraction of EM waves, including the ability to refract light like a medium with a

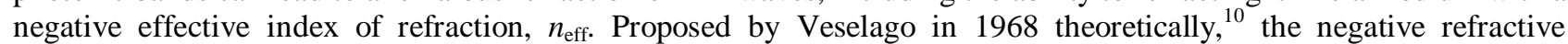
materials were recently become realizable, albeit by an intuitive approach to construct a medium with simultaneously negative permittivity $\varepsilon$ and permeability $\mu$ values. ${ }^{11-14}$ The ability to imitate the $n_{\text {eff }}<0$ without resorting to $\varepsilon<0$, $\mu<0$ created quite an excitement.

In this article, we would like to present and discuss these optical properties of PCs originating from the $n_{\text {eff }}<0$ behavior. In particular, the numerical and experimental demonstration of the negative refraction of EM waves by a photonic crystal slab will be presented. The negative refraction is achieved both for TE and TM polarizations using two different PCs, respectively. The negative refraction then leads to the focusing of EM waves emitted by an omnidirectional source. Here, we demonstrate that the PC based lenses can have superior properties compared to their 
conventional (positive refractive index) counterparts: The PC can provide a focused image with subwavelength resolution, which cannot be obtained using lenses made of ubiquitous materials. Using a different PC, the far field focusing of the EM field through a PC slab can be achieved, which does not depend on a certain optical axis, manifesting true flat lens behavior, and further with a resolution on part with the wavelength. Although the fabrication of PCs working at optical frequencies is still ahead, these results indicate that future PC based lenses can have immaculate imaging capabilities.

This article is organized as follows: In the second section, we investigate the negative refraction and subwavelength focusing in a PC utilizing its TM polarized first band. In the third section negative refraction and focusing analysis of another PC is presented, where, this time, a TE polarized upper band is utilized. We summarize the results in the conclusion part.

\section{NEGATIVE REFRACTION AND SUBWAVELENGTH FOCUSING USING A TM- POLARIZED 2D PHOTONIC CRYSTAL BAND}

The refraction properties of PCs are started to be investigated recently. Kosaka et al. observed an anomalous refraction behavior at the PC interface, which is called as the "super prism phenomenon." ${ }^{15}$ Similar anomalies are reported later by Gralak et al. ${ }^{17}$ Following, Notomi studied the light propagation in strongly modulated PCs theoretically and established the connection between the effective refractive index and the band structure of the PC, by drawing analogies to the effective-mass approximation in electron-band theory. ${ }^{16}$ Luo et al. ${ }^{18}$ investigated all angle negative refraction through a $\mathrm{PC}$, which may act as a point focusing lens.

Analysis of the band structures of PCs revealed the underlying mechanisms for the negative refraction: In one mechanism, the incident field couples to a band with convex equal frequency contours (EFCs) in k-space, where the conservation of the surface parallel component of the wavevector, $\mathrm{k}$, combined with the "negative" curvature of the band causes the incident beam bend negatively. ${ }^{16,}{ }^{18}$ In this case, neither the group velocity nor the effective index is negative and the PC is essentially a positive index medium, exhibiting negative refraction. In another mechanism, if the group velocity and phase velocity derived from the band dispersion are antiparallel for all values of $\mathrm{k}$, the effective index of refraction of the PC becomes negative for that particular frequency range of the band. ${ }^{19,20}$ Both mechanisms are confirmed experimentally. ${ }^{21,22}$ In either case, however, the PC is locally a positive index medium, i.e. $\varepsilon>0$ and $\mu=1$, which distinguishes them from left-handed composite metamaterials. ${ }^{10-14}$

In this section, the experimental and numerical study of negative refraction of TM polarized EM waves through a dielectric PC will be presented. ${ }^{21}$ The PC is a square array of cylindrical alumina rods having dielectric constant $\varepsilon=$ 9.61, diameter $2 r=3.15 \mathrm{~mm}$, and length $l=150 \mathrm{~mm}$. The lattice constant of the PC is $a=4.79 \mathrm{~mm}$. The propagation of the EM wave within the PC can be described by studying the equal frequency contours (EFC) in $k$-space. The transverse magnetic(TM) polarized (i.e., electric field vector, E, is parallel to the rods) first band of the photonic crystal calculated by plane wave expansion method, is shown in Fig. 1.a.

Negative refraction requires convex EFCs for the PC, that are larger than the EFCs for air. [18] The scaled frequency range that gives negative refraction for the present $\mathrm{PC}$ extends from from $\omega=13.10 \mathrm{GHz}$ to $\omega=15.44 \mathrm{GHz}$. The EFCs for air and PC at $\omega=13.698 \mathrm{GHz}$ are shown in Fig. 1.b. Note in the figure that conservation of surface-parallel wave vector gives the direction of the refracted waves inside the PC.

The negative refraction experiment setup consists of an HP 8510C network analyzer, a horn antenna as the transmitter and a monopole antenna as the receiver (Fig. 1.c). The PC used in the refraction measurements has 17 layers in the propagation direction and 21 layers in the lateral direction. The interfaces are along ГM direction. The incident wave makes an angle of $45^{\circ}$ with the normal of the interface. Operating frequency is selected as at $\omega=13.698 \mathrm{GHz}$, since the structure exhibits the maximum angular range of negative refraction at this frequency, as will be discussed later. The spatial distribution of the time averaged incident field intensity along the "front" (air-to-PC) and "back" (PC-to-air) interface locations are measured first in the absence of, and then by placing the PC. For a direct comparison of theoretical predictions and experimental results, simulation of the structure based on experimental parameters using a finite difference time domain (FDTD) method is performed. 


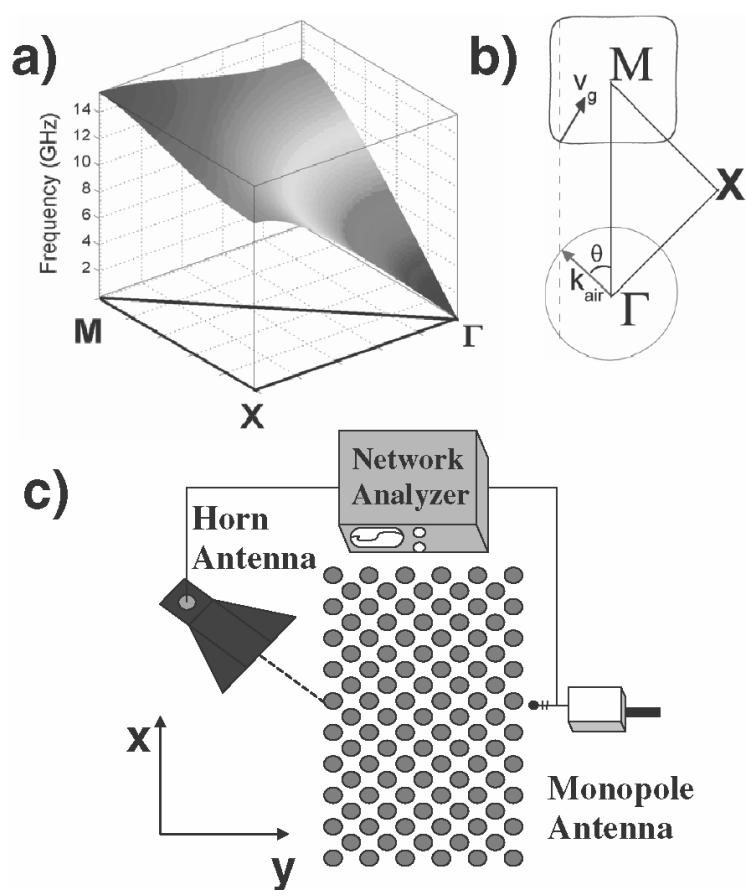

Fig. 1. (a) TM polarized first band of the $2 \mathrm{D}$ cubic photonic crystal. The frequency range in which negative refraction occurs extends from $\omega=13.10 \mathrm{GHz}$ to $\omega=15.44 \mathrm{GHz}$. (b) Frequency contours of air (bottom) and PC (top) at $\omega=13.698 \mathrm{GHz} . . \theta$ denotes the incidence angle from air to PC. (c) Schematic of the experimental. In the refraction experiment, a transmitter horn antenna and a receiver monopole antenna are used. In the focusing effect experiment, both the transmitter and receiver are monopole antennas.

Figure 2.a summarizes the measured and simulated spatial distributions of intensity at the interfaces for the PC. The center-to center shift of the outgoing beam relative to incident beam towards the left side clearly indicates the occurrence of negative refraction. For comparison, Fig. 2.b shows the results for a slab that contains only polystyrene pellets, with a refractive index of 1.46. The refracted beam in this case appears on the right hand side of the incident beam. The positive refractive index determined from the experiment is 1.52 which is close to the tabulated value of 1.46 .

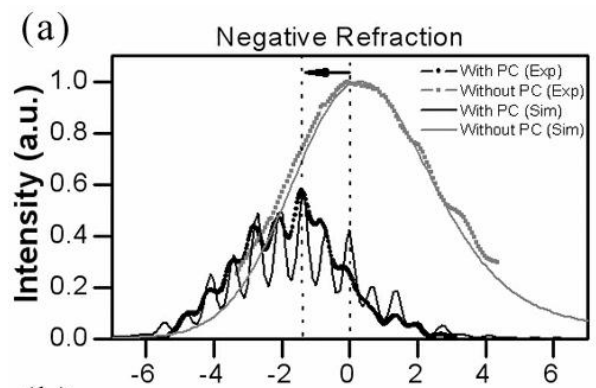

(b)

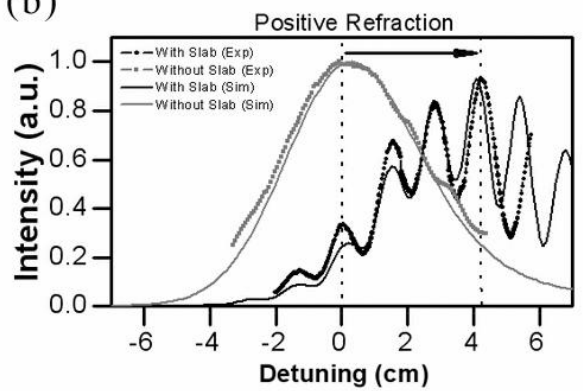

Fig. 2. (a) Refraction through the photonic crystal. Grey dots (grey curve) denote the measured (simulated) average intensity at the at the air-PC interface without PC. Black dots (black curve) denote the measured (simulated) power at the PC-air interface. (b) Same as in (a) but refraction through a crystal of polystryene pellets $(n=1.46)$. 
For the selected frequency, the incident field couples to the first band and propagates according to its dispersion. In the first band, since $\lambda>2 \sqrt{2} a$, the propagation does not suffer from Bragg reflections inside the PC and a well defined single beam propagates through the PC. Hence, Snell's law may be applied:

$$
n_{P C}\left(f, \vec{k}_{i}\right) \sin \left(\theta_{r}\right)=n_{\text {air }} \sin \left(\theta_{i}\right)
$$

where $\theta_{\mathrm{i}}$ is the angle of incidence, $\theta_{\mathrm{r}}$ is the angle of refraction, and $n_{\mathrm{PC}}$ is the effective refractive index of the PC, which depends on frequency and the propagation direction $\vec{k}_{i}$. Based on Eq. 1, $n_{P C}\left(f, \vec{k}_{i}\right)$ determined from the experiment at $\theta_{\mathrm{i}}=45^{\circ}$ is -1.94 , which is very close to the theoretical value of -2.06 calculated by the FDTD method. It is also important to note that the $631 \%$ transmission at this frequency is almost 3 orders of magnitude larger than the typical transmission in a metallic left handed material. ${ }^{11,14}$

In the aforementioned frequency range, the EFCs are square shaped around the $\mathbf{M}$ point in the Brillouin zone (Fig. 1.b). Figure 3 shows the resulting anisotropy of $n\left(\omega, \boldsymbol{k}_{\mathrm{i}}\right)$ at $\omega=13.698 \mathrm{GHz}$ that is determined for various angles of incidence. Negative refraction behaviour is observed for the incidence angles larger than $20^{\circ}$. In this angular range range $\mathbf{v}_{\mathbf{g}} \cdot \mathbf{k}_{\mathbf{i} \|}<0$, (while $\mathbf{v}_{\mathbf{g}} \cdot \mathbf{k}_{\mathbf{i}}>0$ ), where $\mathbf{v}_{\mathbf{g}}$ is the group velocity inside the PC that is given as $\nabla_{k} \omega(k)$ and $\mathbf{k}_{\mathbf{i} \|}$ is the component of wave vector, incident from air to the PC, which is parallel to the interface. At $13.698 \mathrm{GHz}$, the respective EFCs for air and for the PC have almost the same diameter which maximizes the angular range of negative refraction for this structure (Fig. 1.b). If a higher frequency is used, the EFS for air will be larger than the EFS for the PC. In such a case, the maximum angle where we obtain negative refraction gets smaller due to total internal reflection. This results in a narrower angle range for the negative refraction behavior. If a lower frequency is used, we then have EFS for air that is smaller than the EFS for the PC. This in turn increases the minimum angle where we obtain negative refraction, which again reduces the angle range for the negative refraction behavior.

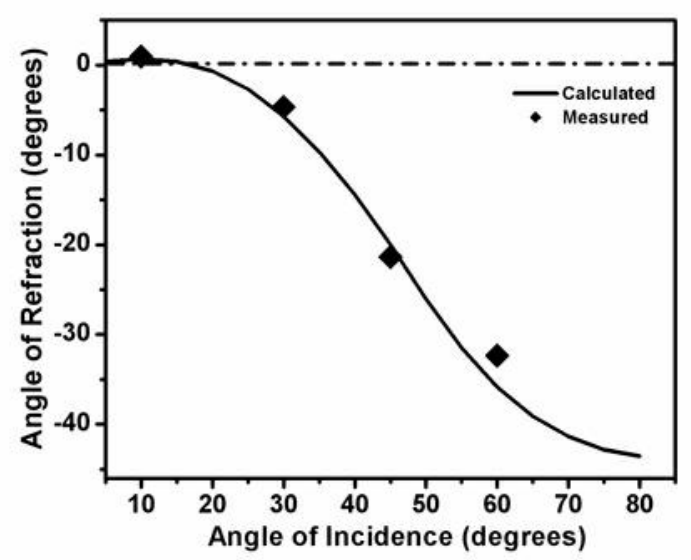

Fig. 3. Anisotropy of the effective index of refraction: Comparison of measured (dots) and simulated (solid curve) angles of refraction versus angles of incidence.

We now discuss the superlensing effect that was proposed first by Pendry, ${ }^{23}$ for negative refractive materials. ${ }^{18,23-25}$ Ideally this requires an isotropic index of refraction of negative unity and amplification of evanescent field modes while propagating across the lens structure which are required for an immaculate image formation on the focal plane. Anisotropy and deviations from negative unity will result in an impaired focusing, but still exhibit unusual focusing 
properties in contrast to positive refractive index materials. The subwavelength resolution, ${ }^{21}$ and image formation ${ }^{22}$ using PC lenses are reported in recent studies.

Since we know the optimum frequency for a broad angle negative refraction, we can use our PC to test the superlensing effect. For a more elaborate investigation of the presence of subwavelength resolution, we introduce two coherent point sources emitting at $\omega=13.698 \mathrm{GHz}$, which are separated by a distance of $\lambda / 3$ and placed $0.7 \mathrm{~mm}$ away from the air-PC interface. Figure 4.a shows the lateral profile of the transmitted power $0.7 \mathrm{~mm}$ away from the PC-air interface obtained from the FDTD simulations (solid lines) and measurements (dots). Evidently the two peaks are clearly resolved in the transmitted signal. The full width at half maximum (FWHM) of the individual image peaks is found to be $0.21 \lambda$. When the crystal is removed, no features are visible on the power profile (dashed line) and the calculated FWHM of the beam at this plane is found to be $5.94 \lambda$. This implies an enhancement of the transmitted field about $25 \times$ compared to free space To exclude interference effects due to coherence, we repeat the experiment using two separate (incoherent) point sources having frequencies $13.698 \mathrm{GHz}$ and $13.608 \mathrm{GHz}$ respectively. Again, the peaks corresponding to the incoherent source pair are clearly resolved (Fig. 4.b).

We stress the point that the narrow incidence angle range for negative refraction $\left(<20^{\circ}\right)$, and the anisotropy restricts the position of the source in the vicinity of the crystal surface for proper observation of focusing effect. The subwavelength imaging is possible due to the amplification of evanescent waves through the PC. ${ }^{25}$ The periodicity of the PC imposes an upper cutoff to the transverse wave vector that can be amplified, which brings an ultimate limit to the superlens resolution. Within this description, we can also introduce an upper limit on the location of the source from the PC. As the evanescent waves have to reach the surface of the PC (before they decay out), the source has to be close enough to the PC. In that sense, the theoretically predicted and the experimentally observed subwavelength resolution in PCs will be limited to the cases where the source is close to the PC.

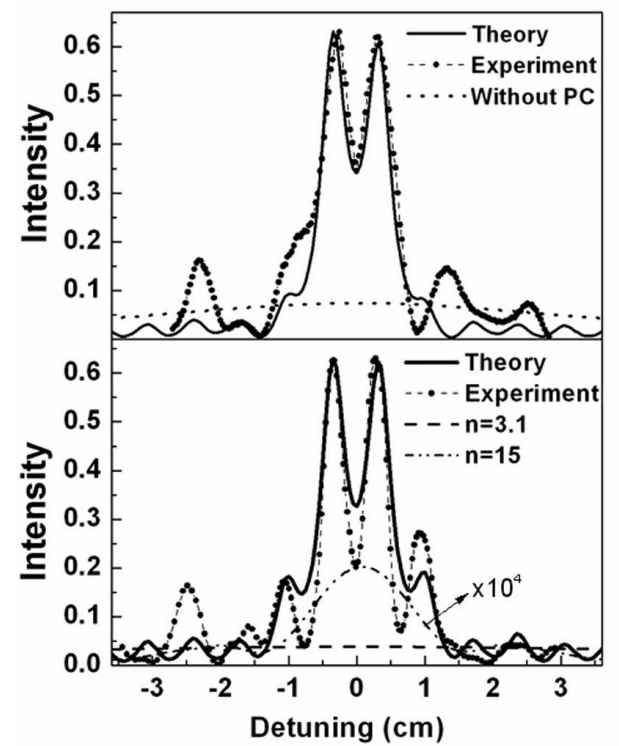

Fig. 4. (a) Measured (dots) and simulated (solid curve) power distribution at $0.7 \mathrm{~mm}$ away from the PC-air interface for two coherent sources separated by $\lambda / 3$ at $0.7 \mathrm{~mm}$ away from the air-PC interface. Dotted line denotes the simulated intensity profile in the absence of PC. (b) Same setup with two incoherent point sources. The lower dashed and dot-dashed curves indicate the power distribution for uniform dielectric slabs with refractive indices $n=3.1$ and $n=15$, respectively. Note the magnification of the $n=15$ curve.

One can argue that the observed enhanced resolution can be attributed to the high refractive index as in the case of oil (or solid) immersion microscopy. In this case, higher wave vectors which are evanescent in air can transmit through the crystal and form a near-field image with subwavelength resolution. In order to check this possibility, the incoherent source setup was simulated for a uniform dielectric slab with a high refractive index. The dashed and dash-dotted lines in Fig. 4.b indicate the power distribution in the presence of a dielectric slab of $n=3.1$ and $n=15$, respectively. The $n$ $=3.1$ uniform slab cannot resolve the sources, and gives a power distribution similar to that in freespace propagation. 
Note that $n=3.1$ is also the refractive index of the alumina rods used in constructing the PC. Even with the use of artificially high refractive index $(n=15)$, the sources are not resolved. Besides, the large reflection due to high index contrast at the interface significantly reduces the transmitted power when compared to the high $(63 \%)$ transmission obtained from the PC at this operating frequency. So, even if the observed subwavelength is associated with near field effects, this is not achievable by ubiquitous materials.

\section{SPECTRAL NEGATIVE REFRACTION AND FOCUSING ANALYSIS OF THE TE POLARIZED BAND OF A 2D PHOTONIC CRYSTAL}

In the previous section, the negative refraction and focusing effect originating from the convex equal frequency contours of the first band are investigated. Although the focusing abilities surpasses that of conventional lenses, both the frequency range and the angular range for the occurrence of negative refraction is limited. In this section, we employ a different band topology of a 2D photonic crystal to obtain negative refraction. Our aim is to achieve a negative $n_{\text {eff }}$ with higher isotropy. Based on the analysis presented in Ref. [19], we utilize a TE polarized upper band of the PC (i.e., the magnetic field $\boldsymbol{H}$ is parallel to the dielectric rods).

The PC is a hexagonal lattice of alumina rods in air with a lattice period of of $a=4.79 \mathrm{~mm}$. The rods have dielectric constant $\varepsilon=9.61$, diameter $2 r=3.15 \mathrm{~mm}$, and length $l=15 \mathrm{~cm}$. Figure 5.a shows the transverse electric (TE) polarized band structure in the first Brillouin zone. The $5^{\text {th }}$ band shaded in the figure extends from $\tilde{\omega}=0.65(\omega=40.65 \mathrm{GHz})$ to $\widetilde{\omega}=0.74(\omega=46.27 \mathrm{GHz})$, where $\widetilde{\omega}=\omega a / 2 \pi c$ is the scaled frequency. In Fig. 5.b, , the band surface in the full Brilloin zone along with the projected equal frequency contours (EFCs) are shown. The EFCs of the band are shrinking with increasing frequency, contrary to the EFCs in air $(n=1)$ which are given by the dispersion $\omega=c k$. As a result, the effective refractive index $n_{p}=\operatorname{sgn}\left(\vec{v}_{g} \cdot \vec{k}_{f}\right)\left(c\left|\vec{k}_{f}\right| / \omega\right)$, becomes negative due to sign of the antiparallel group velocity $\vec{v}_{g}=\nabla_{\vec{k}} \omega$ and the phase velocity $\vec{v}_{p}=\left(c /\left|n_{p}\right|\right) \hat{k}_{f}$. Here $c$ is the speed of light in vacuum, and $\hat{k}_{f}$ is the unit wavevector in the PC. We note that the EFCs start to deviate from the circular shape towards the bottom edge of the band, which will induce some anisotropy. We will discuss this effect later.

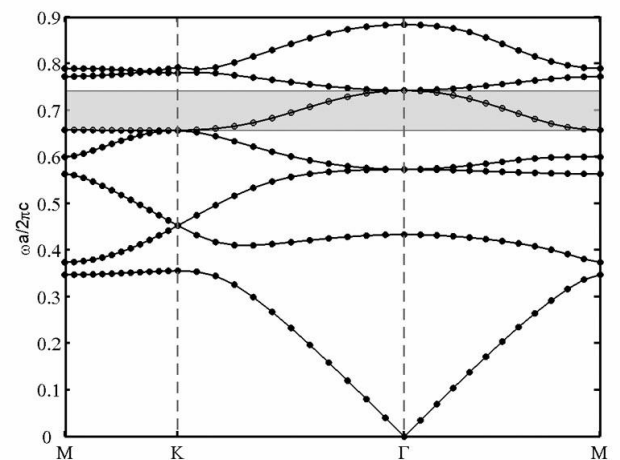

(a)

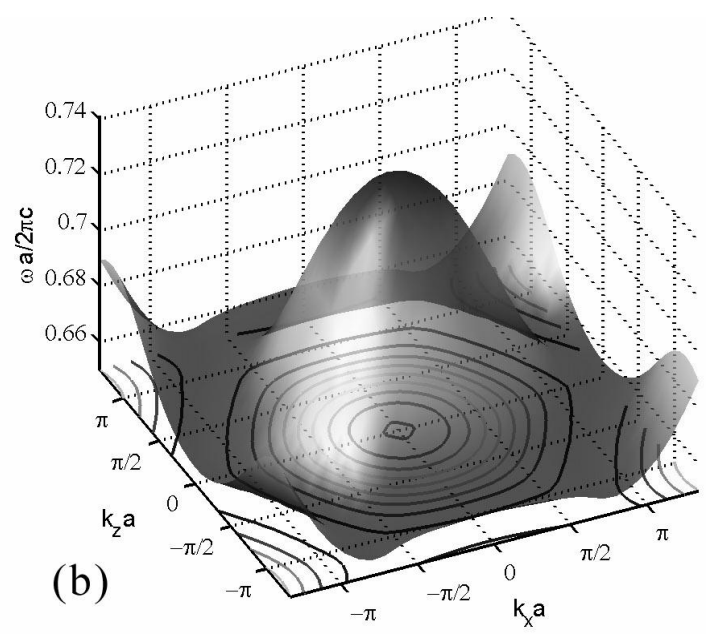

Fig. 5. (a) TE polarized band structure of the hexagonal PC. The 5th band (shaded) has negative effective index of refraction. (b) The surface (transparent shell) of the 5th band in the full Brillouin zone. Some equal frequency contours are projected to the Brillouin zone plane.

The refraction spectra are measured by a setup consisting of a network analyzer, a microwave horn antenna as the transmitter and a waveguide antenna as the receiver. The PC has 7 layers along the incidence ( $\Gamma \mathrm{M})$ direction and 31 layers along the lateral $(\Gamma \mathrm{K})$ direction. The horn antenna is located on the negative side of the PC with respect to its central axis. The spatial intensity distribution along the PC-air interface is scanned in $1.27 \mathrm{~mm}$ steps, while the 
frequency is swept from $38.5 \mathrm{GHz}$ to $43.5 \mathrm{GHz}$. Figure 6(a) displays the transmission spectra as a function of frequency and lateral position for three different incidence angles of $\theta_{i}=15^{\circ}, 30^{\circ}$, and $45^{\circ}$. It is evident that the transmitted beam appears on the negative side. We observe that the anisotropy due to frequency dependence causes some spatial broadening. When the incidence angle is increased, the transmission shifts towards left accordingly. To investigate the beam profiles, the spatial cross section at $\omega=41.7 \mathrm{GHz}(\tilde{\omega}=0.667)$ are plotted in Fig. $6(\mathrm{~b})$. We remind the reader that the incident field has a Gaussian beam profile centered at $x=0$ (not shown on figures). The intensities are normalized with respect to the maximum intensity for the $15^{\circ}$ incidence. It is apparent that the lateral shift is accompanied by a decrease in the transmission intensity. This is mainly due to the increase of reflection with increasing incidence angle. Another contribution is the diffraction induced out-of-plane (y-direction) loss within the PC. Figure 6.(c) displays the corresponding profiles obtained from FDTD simulations showing very good agreement with measurements.
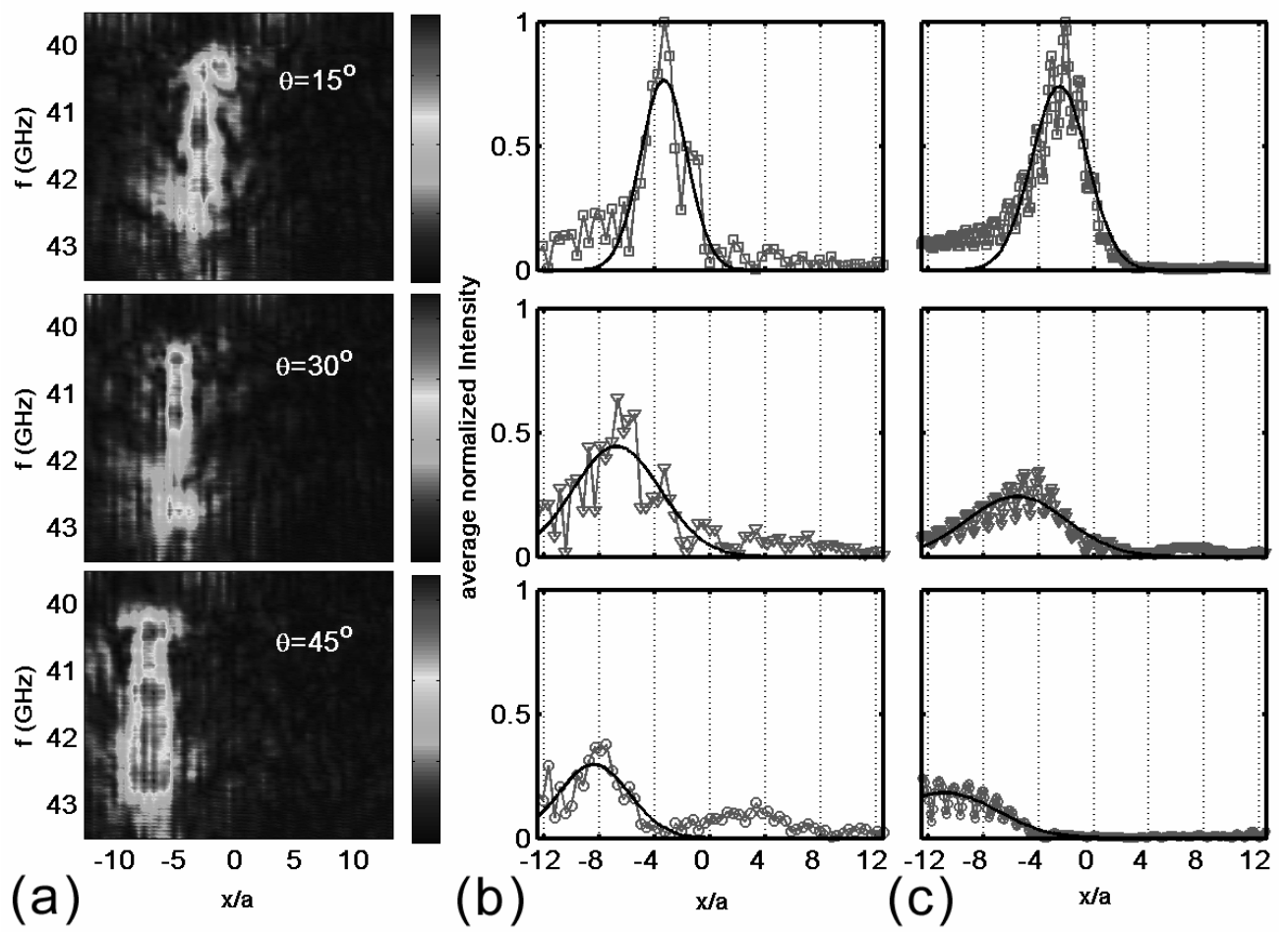

Fig. 6. (a) Measured negative refraction spectra of the 5th band along the PC-air interface for incidence angles $\theta_{i}=15^{\circ}, 30^{\circ}$, and $45^{\circ}$. Measured (b) and simulated (c) intensity profiles at $\omega=41.7 \mathrm{GHz}$ for the respective incidence angles. Solid curves indicate Gaussian fits.

In order to gain insight about the propagating field within the crystal, we have performed an FDTD simulation for the $\theta_{i}=30^{\circ}$ incidence, shown in Fig. 7. Evidently, higher order reflection (marked 3) is present because at this frequency

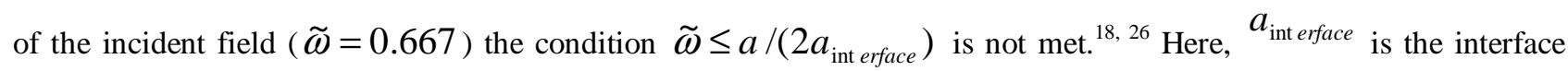
period of the PC. The transmitted beam propagating as a single component on the other side of the PC (marked 5) indicates that the refracted beam within the PC (marked 4) can be regarded as single component in the sense that most of the propagating power is coupled to the zero-order diffracted wave, and Snell's law is applicable. Previous studies also pointed out that single beam refraction at higher bands is possible. ${ }^{16,20,26}$ 


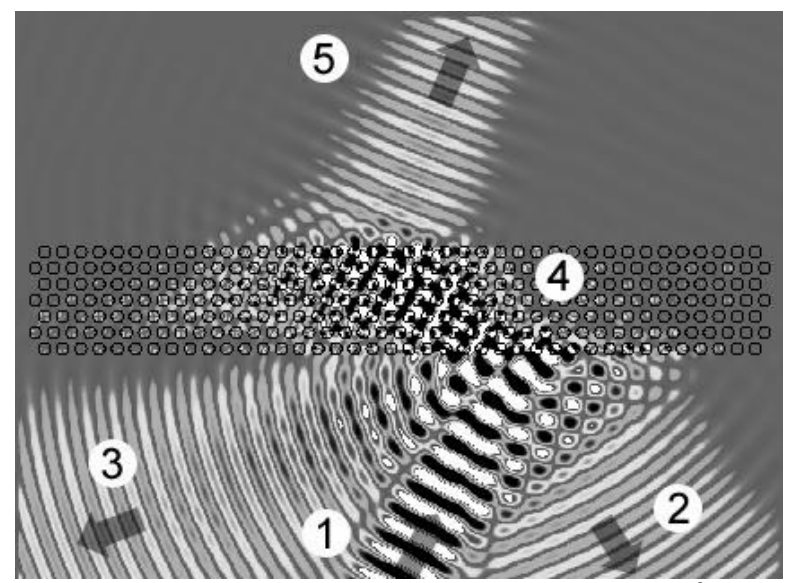

Fig. 7. Simulated negative refraction of a plane wave at $\omega=41.7 \mathrm{GHz}$ incident at $\theta_{\mathrm{i}}=30^{\circ}$ to the PC interface (mark 1). Zero order (mark 2) and higher order (mark 3) reflections occur. Despite the complicated wave pattern composed of Bloch modes within the PC (mark 4), the transmitted field (mark 5) propagates as a single beam.

Using Snell's law for this geometry by $n\left(\omega, k_{i}\right) \sin \theta_{f}=n_{\text {air }} \sin \theta_{i}$, where $\theta_{i}$ is the angle of incidence and $\theta_{f}$ is the angle of refraction inside the PC, we obtain from the experiment $n_{\text {eff }}=-0.52,-0.66$, and -0.86 for $\theta_{i}=15^{\circ}, 30^{\circ}$, and $45^{\circ}$, respectively. The simulation results for the same incidence angles give $n_{\text {eff }}=-0.66,-0.72$, and -0.80 , respectively. The anisotropy of the effective refractive index is evident from these figures. We note that the operating frequency 41.7 $\mathrm{GHz}$ for which the lateral beam profiles are plotted is rather close to the lower edge of the band, which makes this anisotropy more prominent. We stress that the frequency range $(40.65-43 \mathrm{GHz})$ plotted in Fig. 6.a covers only the lower half of the band. The range above $43 \mathrm{GHz}$ was beyond the operation limit of our measurement setup. Obviously, $n_{\mathrm{eff}}$ of the band should change with frequency since it should approach to zero at the upper band edge.

In order to investigate the focusing properties of this PC, we first performed FDTD simulations for a TE polarized point source at $\omega=42.07 \mathrm{GHz}$, located at a distance $d_{\mathrm{src}}=2 \lambda$ away from the air-PC interface. Figure 8.(b) shows the resulting normalized spatial intensity distribution in the image plane. The PC-air interface is located at $z=0$. The peak indicates the focusing behaviour unambiguously (we also refer the reader to Fig. 10.a for the simulated 2D field map, where the convergence of wavefronts to a focal point along the optical axis is clearly visible). We would like to emphasize that the focusing occurs away from the PC-air interface, at $\mathrm{z} \approx 8 \lambda$. The significant aberration of the focus pattern along the propagation axis can be attributed to the fact that $n_{\text {eff }}$ deviates from negative unity, and bears certain amount of anisotropy as discussed above. We note that even when $n_{\text {eff }}$ were perfectly isotropic and uniform, a value different than negative unity would not generate perfect point focusing. Therefore, unlike the focusing discussed in the previous section, this PC does not perform "imaging" in a strict sense. On the other hand, focusing effects induced by channelling may be excluded, which occur close to the interface. In the subwavelength focusing studies reported previously, the source is located in the vicinity of the PC interface $\left(d_{\mathrm{src}}<\lambda\right){ }^{21,}{ }^{22}$ There are also studies concerning the focusing of Gaussian beams using PC structures in the microwave, and infra-red regimes. ${ }^{27,28}$

In the experiment, a waveguide aperture is used as the source, and the intensity distribution in the image plane is measured by a monopole antenna. For $d_{\mathrm{src}}=2 \lambda$, first the propagation direction, $z$, is scanned for locating the maximum intensity, and then lateral cross sections of intensity at several $z$ around the peak position are measured. In Fig. 8.a the focusing of the beam both in lateral and longitudinal directions is evident. The maximum intensity (normalized to unity) is observed at $d_{\text {focus }} / \lambda \approx 8$. We note the similarity of the measured and simulated focusing profiles. 

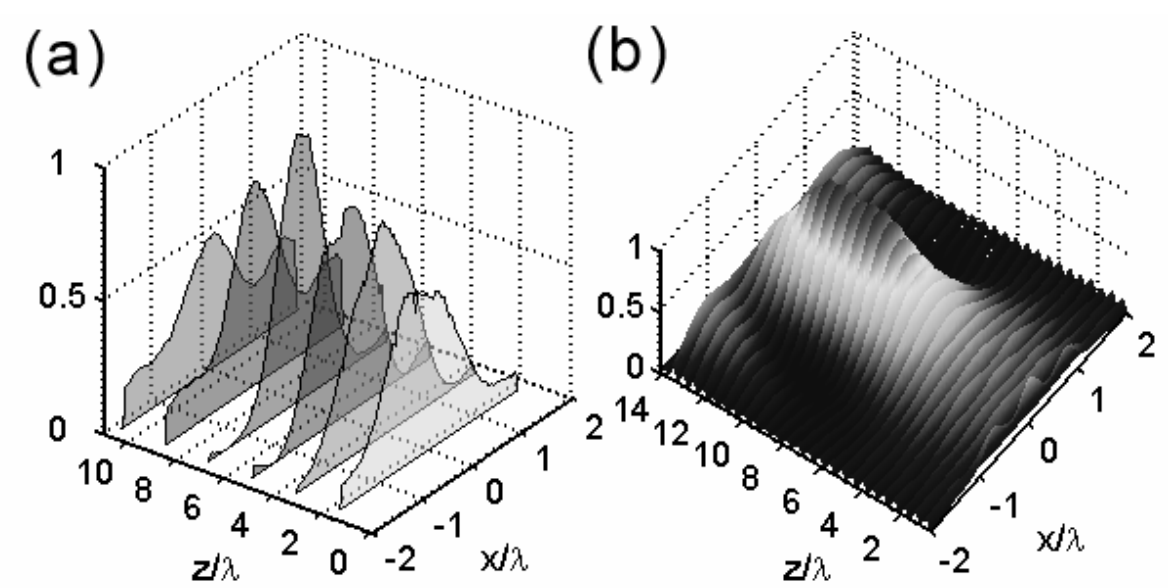

Fig. 8. (a) Lateral intensity profiles measured at six different positions along the propagation direction: $z / \lambda=1.78,3.56,5.34,7.12$, 8.90, and 10.68. $z=0$ corresponds to the PC interface. Source is located at $z / \lambda=2$ away from the other interface (not shown in figure) (b) The corresponding FDTD simulated 2D magnetic field intensity in the image plane.

The flat lens behaviour of the PC structures was emphasized in recent studies. ${ }^{22}$ In this case, the focusing properties are preserved at an arbitrary lateral location of the source along the interface. To demonstrate this, we have shifted the source laterally and measured the lateral beam profile at the focal point. In Figure 9, the source positions (vertical dashed lines) and the peak positions of the focused beam (symbols) are in excellent agreement. The agreement is supported by the simulated profiles (solid curves) using a true point source. The average FWHM of the profiles at $\mathrm{d}_{-}$focus $\approx 8 \lambda$ depicted in Fig. 9 is $1.60 \mathrm{a} \approx 1.08 \lambda$, on par with the wavelength.

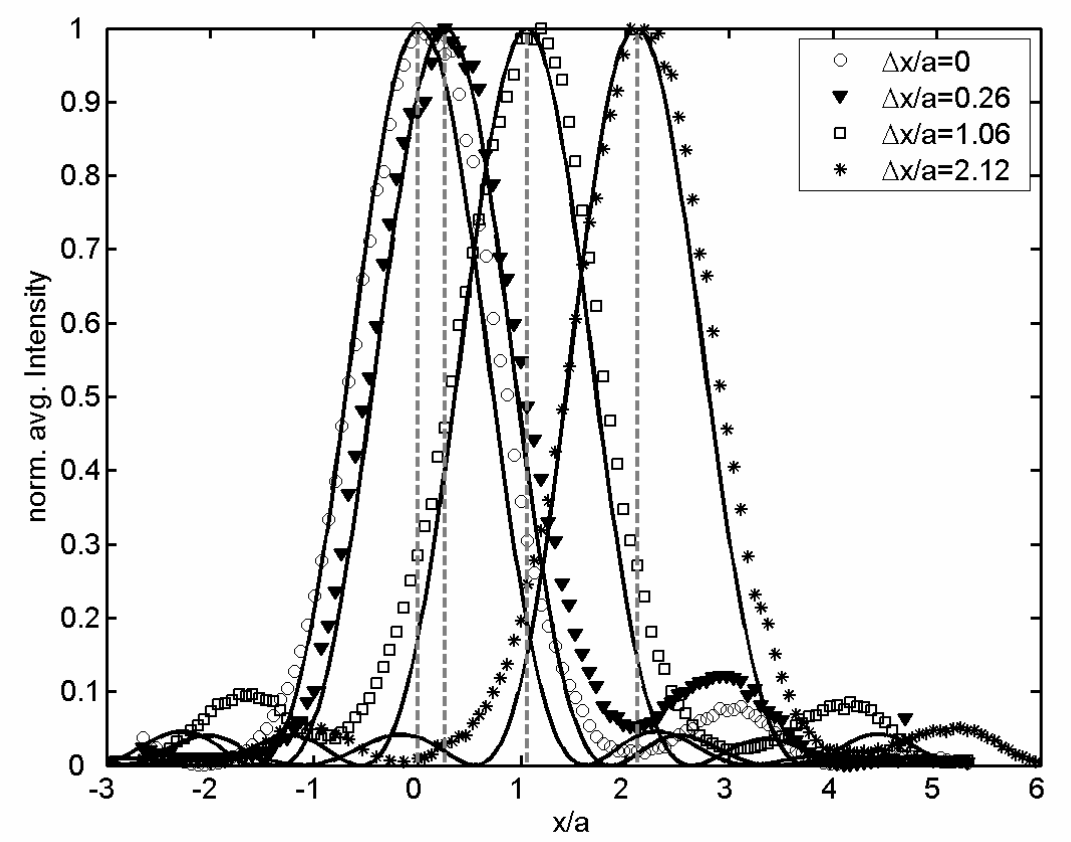

Fig. 9. The measured (symbols) and simulated (solid curves) lateral intensity profiles at the focal distance $z / \lambda \approx 8$ for different lateral shifts. The respective source locations are denoted by vertical dashed lines.

We further investigated the effect of shifting the source towards and away from the PC interface. The FDTD simulated 2D map of the magnetic field, $\mathbf{H}(x, z)$, plotted in Fig. 10.a shows that when the source is moved from $d_{\text {src }}=2 \lambda$ to $d_{\text {src }}=$ $4 \lambda$ away, the focus pattern on the other side shifts accordingly towards the PC-air interface. In Figure 10.b, the measured 
lateral profiles at focus points for various $d_{\text {src }}$ are plotted. From the determined focal positions we have found that $d_{\text {src }}+$ $d_{\text {focus }}$ remains roughly constant, which may be investigated further within a geometric optics analysis. The figure also displays the intensity profile in the absence of the PC for $d_{\mathrm{src}}=2 \lambda$ case (dotted line) which shows almost no features about the source location. Overall, the focused beam by the $\mathrm{PC}$ is $\sim 12 \mathrm{~dB}$ higher relative to freespace propagation at the focal distance and has a FWHM of the order $\lambda$.
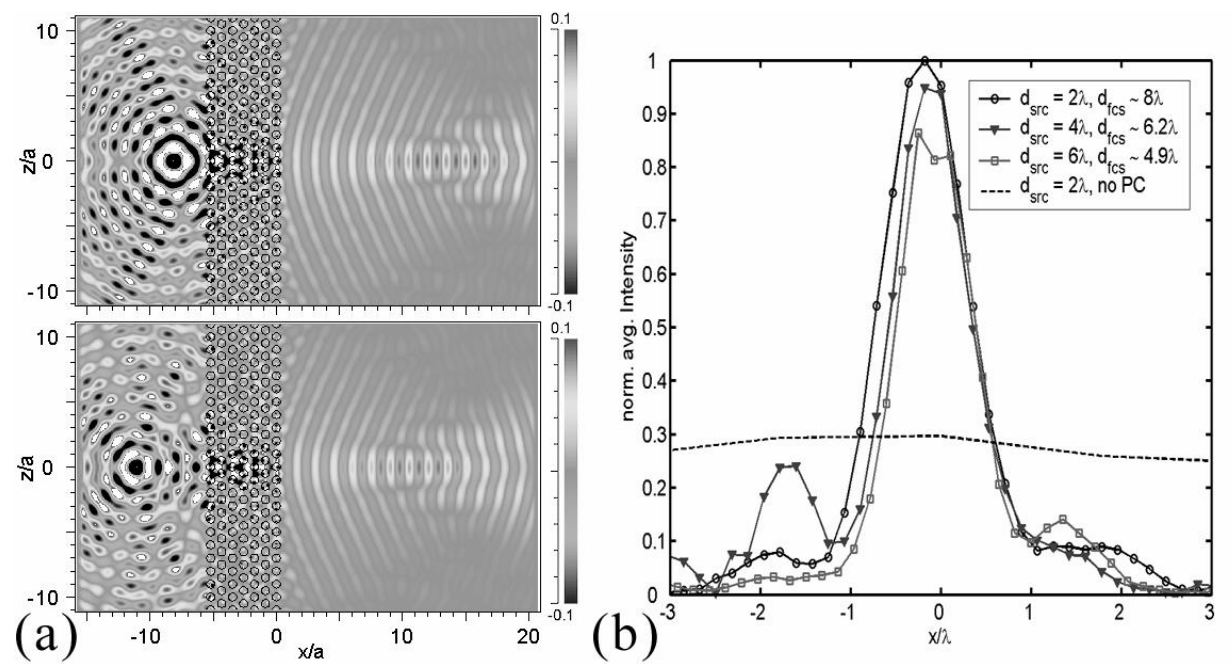

Fig. 9. (a) The FDTD simulation of the $2 \mathrm{D}$ magnetic field distribution $\mathbf{H}(x, z)$ for $d_{\mathrm{src}}=2 \lambda$ (top) and $d_{\mathrm{src}}=4 \lambda$ (bottom). (b) The measured lateral intensity profiles at respective focal distances for different source distances. The intensity is normalized by the $d_{\text {src }}=$ $2 \lambda$ profile peak. The dashed line denotes the intensity profile in the absence of PC for $d_{\text {src }}=2 \lambda$.

\section{CONCLUSION}

Photonic crystals bring profound changes and extensions to our ability of shaping the propagation of EM waves. In this article, we have presented the negative refraction and the focusing abilities of 2D dielectric photonic crystals. An effective index of refraction, $n_{\text {eff }}$, can be defined from the band structure of the PC, which, under convex EFCs, can take negative values and can be associated with refraction of electromagnetic waves through the PC. Consequently, the details of the band structure are inherited in the (an)isotropy of $n_{\text {eff. Nevertheless, the focusing }}$ abilities associated with $n_{\text {eff }}$ are promising: Both the subwavelength resolution and far field focusing is achievable using a PC with appropriately chosen parameters. A simple rectangular PC slab can exhibit true flat lens behavior (i.e., absence of an optical axis) which makes it simple and robust optical element both from fabrication and applications points of view.

It is important to emphasize that the electromagnetic phenomena demonstrated here depends essentially on the refractive index of the dielectric material and on the geometrical parameters of the 2D PC only. Consequently, scaling the PC can bring this phenomena across the electromagnetic spectrum to any relevant regime, provided that the fabrication techniques retain the uniformity of the structure. This makes the PCs potentially available for a very wide range of photonic and electromagnetic applications.

\section{REFERENCES}

1. J. D. Joannopoulos, R. D. Meade, and J. N. Winn, Photonic Crystals: Molding the Flow of Light, Princeton University Press, Princeton, 1995.

2. C. M. Soukoulis (ed.), Photonic Crystals and Light Localization in the 21st Century, Kluwer, Dortrecht, 2000.

3. H. Benisty, C. Weisbuch, D. Labilloy, M. Rattier, C. J. M. Smith, T. F. Krauss, R. M. De La Rue, R. Houdré, U. Oesterle, C. Jouanin, and D. Cassagne, J. Lightwave Tech. 17, p.2063, 1999.

4. T. Krauss, Y. P. Song, S. Thomson, C. D. Wilkinson, and R. M. De La Rue, Electron. Lett. 30, p. $1444,1994$. 
5. P. L. Gourley, J. R. Wendt, G. A. Vawter, T. M. Brennan, and B. E. Hammons, Appl. Phys. Lett. 64, p.687, 1994.

6. S. Noda, M. Imada, S. Ogawa, M. Mochizuki, and A. Chutinan, IEEE Jour. Quant. Elec. 38, p. $726,2002$.

7. J. M. Lourtioz, H. Benisty, A. Chelnokov, S. David, and S. Olivier, Annals of Telecommunications 58, p. 1197, 2003.

8. E. Kuramochi, M. Notomi, T. Kawashima, C. Takahashi, J. Takahashi, T. Tamamura, and S. Kawakami, Proc. Int. Workshop of Photonic and Elect. Crystal Struct. (PECS II), Sendai, Japan, 2000.

9. Y. Sugimoto, N. Ikeda, N. Carlsson, K. Asakawa, N. Kawai, and K. Inoue, J. Appl. Phys. 91, p. $922,2002$.

10. V. G. Veselago, Soviet Physics Uspekhi 10, p. 509, 1968.

11. D. R. Smith, W. J. Padilla, D. C. Vier, S. C. Nemat-Nasser, and S. Schultz, Phys. Rev. Lett. 84, p. 4184, 2000.

12. R. A. Shelby, D. R. Smith, S. C. Nemat-Nasser, and S. Schultz, Appl. Phys. Lett. 78, p. 481, 2001.

13. R. A. Shelby, D. R. Smith, and S. Schultz, Science 292, p. 77, 2001.

14. M. Bayindir, K. Aydin, E. Ozbay, P. Markos, and C. M. Soukoulis, Appl. Phys. Lett. 81, p. 120, 2002.

15. H. Kosaka, T. Kawashima, A. Tomita, M. Notomi, T. Tamamura, T. Sato, and S. Kawakami, Phys. Rev. B 58, p. R10096, 1998.

16. M. Notomi, Phys. Rev. B 62, p. 10696, 2000.

17. B. Gralak, S. Enoch, and G. Tayeb, J. Opt. Soc. Am. A 17, p. 1012, 2000.

18. C. Luo, S. G. Johnson, J. D. Joannopoulos, and J. B. Pendry, Phys. Rev. B 65, p. R201104, 2002.

19. S. Foteinopoulou, E. N. Economou, and C. M. Soukoulis, Phys. Rev. Lett. 90, p. 107402, 2003.

20. S. Foteinopoulou, and C. M. Soukoulis, Phys. Rev. B 67, p. 235107, 2003.

21. E. Cubukcu, K. Aydin, E. Ozbay, S. Foteinopoulou, and C. M. Soukoulis, Nature 423, p. 604, 2003; Phys. Rev. Lett. 91, p. 207401, 2003.

22. P. V. Parimi, T. W. Lu, P. Vodo, and S. Sridhar, Nature 426, p. 404, 2003.

23. J. B. Pendry, Phys. Rev. Lett. 85, p. 3966, 2000.

24. R. W. Ziolkowski, and E. Heynman, Phys. Rev. E 64, p. 056625, 2001.

25. C. Luo, S. G. Johnson, J. D. Joannopoulos, and J. B. Pendry, Phys. Rev. B 68, p. 045115, 2003.

26. S. Foteinopoulou, and C. M. Soukoulis, http://arxiv.org/cond-mat/0403542, 2004.

27. A. Martínez, H. Míguez, A. Griol, and J. Martí, Phys. Rev. B 69, p. 165119, 2004.

28. A. Berrier, M. Mullot, M. Swillo, M. Qiu, L. Thylén, A. Talneau, and S. Anand, Phys. Rev. Lett. 93, p. 073902, 2004.

29. Zhi-Yuan Li and Lan-Lan Lin, Phys. Rev. B 68, p. 245110, 2003. 